\title{
Efficient Method for Calculating the Composite Stiffness of Parabolic Leaf Springs with Variable Stiffness for Vehicle Rear Suspension
}

\author{
Wen-ku Shi, Cheng Liu, Zhi-yong Chen, Wei He, and Qing-hua Zu \\ State Key Laboratory of Automotive Simulation and Control, Jilin University, Changchun 130025, China \\ Correspondence should be addressed to Zhi-yong Chen; chen_zy@jlu.edu.cn
}

Received 7 October 2015; Revised 28 January 2016; Accepted 2 February 2016

Academic Editor: Reza Jazar

Copyright (C) 2016 Wen-ku Shi et al. This is an open access article distributed under the Creative Commons Attribution License, which permits unrestricted use, distribution, and reproduction in any medium, provided the original work is properly cited.

\begin{abstract}
The composite stiffness of parabolic leaf springs with variable stiffness is difficult to calculate using traditional integral equations. Numerical integration or FEA may be used but will require computer-aided software and long calculation times. An efficient method for calculating the composite stiffness of parabolic leaf springs with variable stiffness is developed and evaluated to reduce the complexity of calculation and shorten the calculation time. A simplified model for double-leaf springs with variable stiffness is built, and a composite stiffness calculation method for the model is derived using displacement superposition and material deformation continuity. The proposed method can be applied on triple-leaf and multileaf springs. The accuracy of the calculation method is verified by the rig test and FEA analysis. Finally, several parameters that should be considered during the design process of springs are discussed. The rig test and FEA analytical results indicate that the calculated results are acceptable. The proposed method can provide guidance for the design and production of parabolic leaf springs with variable stiffness. The composite stiffness of the leaf spring can be calculated quickly and accurately when the basic parameters of the leaf spring are known.
\end{abstract}

\section{Introduction}

The development of lightweight technology and energy conservation has resulted in the extensive application of leaf springs with variable stiffness on cars. Leaf springs with variable stiffness are one of the focal points in automobile leaf springs because of their advantages over traditional leaf springs [1-5]. Parabolic leaf springs are springs that have leaves with constant widths but have varying cross-sectional thicknesses along the longitudinal direction following a parabolic law. This type of leaf spring has many advantages, such as lightness of weight; mono-leaf springs have been applied in car parts. However, the reliability of multileaf springs cannot be fully guaranteed because of manufacturing limitations; therefore, this type of spring has not been widely applied on cars. Double-leaf parabolic springs with variable stiffness are the simplest form of multileaf springs with mono-leaf springs being applied in car parts. However, the reliability of multileaf springs cannot be fully guaranteed because of manufacturing limitations; therefore, this type of spring has not been widely applied on cars. Double-leaf parabolic springs with variable stiffness are the simplest form of multileaf springs with variable stiffness and are also the final generation of this type of multileaf spring. At present, a second main spring is added under the first main spring to protect the latter for safety considerations, thus forming a triple-leaf spring with variable stiffness.

Stiffness is an important design parameter for leaf springs with variable stiffness. This parameter can be calculated using three methods, namely, formula method, FEA method, and rig test. The formula and FEA methods are preferred over the rig test because of the high manpower and time requirements of the latter. The formula method is commonly used in calculating leaf spring stiffness. In [1], the main and auxiliary springs are modeled as multileaf cantilever beams, and an efficient method for calculating the nonlinear stiffness of a progressive multileaf spring is developed and evaluated. In [2], the stiffness of the leaf spring is calculated by an in-house software based on mathematical calculations using the thickness profile of the leaves. In [3], the deformations of the main 


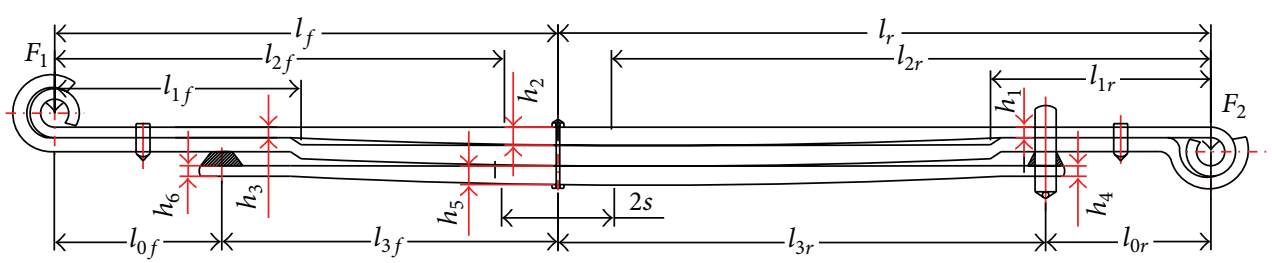

FIgURE 1: Two levels of parabolic leaf spring with variable stiffness.

and auxiliary springs are considered simultaneously to calculate the stiffness of the leaf spring with variable stiffness by the method of common curvature. In [4], a force model of one type of leaf spring with variable stiffness is established, and a curvature-force hybrid method for calculating the properties of such a leaf spring is developed. In $[5,6]$, an equation for calculating the stiffness of a leaf spring with large deflection is derived. The FEA method is also frequently used to calculate the leaf spring stiffness. In [7-9], leaf spring stiffness is calculated using the FEA method, and the result is verified by a rig test. In [10], the finite element method was used to calculate the stiffness of the parabolic leaf spring, and the effects of materials, dimensions, load, and other factors on the stiffness are discussed. In [11], an explicit nonlinear finite element geometric analysis of parabolic leaf springs under various loads is performed. The vertical stiffness, wind-up stiffness, and roll stiffness of the spring are also calculated. The FEA method is more commonly used than the formula method in the calculation of leaf spring stiffness. However, the former is more complex than the latter and requires specialized finite element software as a secondary step. Engineers who use this method should also have some work experience. By contrast, designers can easily master the formula method in calculating the stiffness of the leaf spring because it is simple and does not require special software and computer aid. Thus, deriving a stiffness calculation formula for leaf springs is significant. However, the type and size of leaf springs vary. Thus, no uniform formula can be applied to all types of leaf springs. Various equations can only fit a particular type of leaf spring. Current studies on calculating the stiffness of parabolic leaf springs with variable stiffness mostly considered the FEA method. Hence, a simple composite stiffness calculation formula should be derived for this method.

The main spring bears the load alone when the load is small in parabolic leaf springs with variable stiffness in the form of main and auxiliary springs. Thereafter, the main and auxiliary springs come into contact because of the increased load. Finally, the main and auxiliary springs bear the load together when the main and auxiliary springs are under full contact. Therefore, the stiffness of the spring changes in three stages. During the first stage, the stiffness of the spring is equal to the stiffness of the main spring before the main spring and secondary springs come into contact. During the second stage, stiffness increases when the main and auxiliary springs come into contact. During the third stage, the stiffness of the spring has a composite stiffness generated by both the main and auxiliary springs when the springs are under full contact.
Changes in the stiffness of the leaf spring during stage two are nonlinear, and the stiffness is difficult to calculate. Three calculation equations are currently used [12-14] to calculate the stiffness of mono-leaf parabolic springs. However, only integral equations are available for calculating the composite stiffness of parabolic leaf spring with variable stiffness [12, 13]. However, the direct calculation of the composite stiffness of a taper-leaf spring using integral equations is quite challenging, and these equations cannot be applied during the design of a leaf spring. In this paper, a simple and practical equation for calculating the composite stiffness of parabolic leaf springs with variable stiffness is derived. The equation can be used not only for calculating the composite stiffness but also for designing such a type of leaf spring.

In this paper, firstly, the leaf spring assembly model was simplified. A simple model of double-leaf parabolic spring with variable stiffness (referred to as double-leaf spring model) was considered. The difficulties in the calculation of the composite stiffness of double-leaf spring were analyzed, and the equation for calculating the composite stiffness was derived using the method of material mechanics. Thereafter, the superposition principle was used to derive the equation for triple-leaf springs. This equation can be extended to calculate the stiffness of multileaf springs. The correctness of the equation was verified by a rig test and finite element simulation. Finally, the parameters that should be considered when using the equations for leaf spring design were discussed.

\section{Model of Two-Level Parabolic Leaf Spring with Variable Stiffness and Its Simplification}

2.1. Leaf Spring Assembly Model. The leaf spring studied in this paper is a parabolic leaf spring with variable stiffness and unequal arm length. A triple-leaf spring model is analyzed (Figure 1). The triple-leaf spring consists of two main springs and an auxiliary spring. The stiffness of the spring is designed as a two-level variable stiffness after the requirements for a comfortable ride under different loads were considered. The first-level stiffness is the stiffness of the main spring, and the second-level stiffness is a composite stiffness determined by the stiffness of both the main and the auxiliary springs. The rear shackle is designed as an underneath shackle that makes the length of the auxiliary spring significantly less than that of the main spring because of the limited installation space 
for the spring, security (leaf spring reverse bend), and vehicle performance requirements. Thus, the composite stiffness of the multileaf spring is not a simple stiffness superposition of multiple parabolic spring leaves with equal lengths.

2.2. Model Simplification. The composition of the structure of the parabolic leaf spring should be analyzed first to study its stiffness. The two main springs have the same widths and thicknesses. The primary function of the second main spring is to protect the first main spring, increasing the reliability of the leaf spring. The spring leaves contact by end, and an antifriction material is placed at the contact area. Thus, interleaf friction is not considered in the simplified model. The leaf spring force model can be simplified to a cantilever model according to the theory of material mechanics (Figure 2). The blue dashed line in Figure 2 shows an imaginary parabolic portion of the leaf spring to demonstrate the cross-sectional shape variation law of parabolic leaf springs. The triple-leaf spring model in this paper is a combination of a monoleaf (Figure 3) and a double-leaf spring model (Figure 4). The stiffness of the mono-leaf spring model can be easily obtained; thus, this study focuses on calculating the stiffness of the model shown in Figure 4. The model of a two-level leaf spring with variable stiffness will eventually develop into a double-leaf spring model (Figure 4). The highest utilization efficiency of the leaf spring material and lowest assembly weight are considered.

Several assumptions are developed according to the theory of material mechanics as follows:

(1) The leaf spring is generally considered a cantilever beam. A fixed constraint is applied at one of the ends. A concentration force perpendicular to the surface of the main spring is applied at the other end.

(2) The unique type of force between the leaves is a vertical force.

(3) The boundaries between the main and auxiliary springs should have the same curvatures and deflections.

(4) The thickness of the leaves is negligible, and the main and auxiliary springs are in full contact.

\section{Derivation of Equation for Calculating the Composite Stiffness for Two-Level Parabolic Leaf Springs with Variable Thickness}

A model for the parabolic double-leaf spring with variable stiffness (referred to as double-leaf spring model) is first examined according to the theory in Section 2. Thereafter, according to the theory of superimposition, a triple-leaf spring model was studied. Finally, the results are applied to a multileaf spring.

3.1. Challenges in Composite Stiffness Calculation. Various types of equations are used to calculate the composite stiffness of parabolic leaf springs with variable stiffness. The design part of the automobile engineering manual [7] and Spring Manual

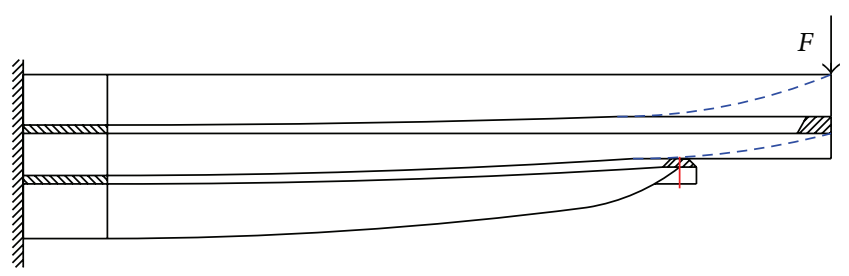

Figure 2: Cantilever beam model showing the two levels of the parabolic leaf spring with variable stiffness.

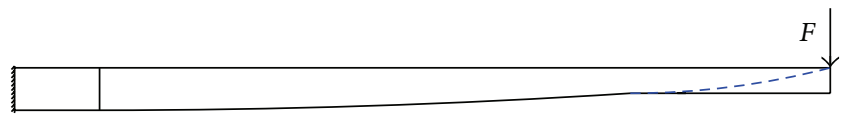

Figure 3: Cantilever beam model of a parabolic mono-leaf spring.

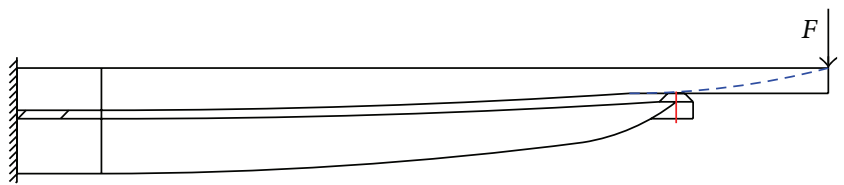

FIGURE 4: Cantilever beam model of a parabolic double-leaf spring with variable stiffness.

[8] provide the following integral equation for calculating composite stiffness:

$$
\frac{1}{K}=\frac{1}{2 \xi} \int_{0}^{l_{m}} \frac{x^{2}}{E\left(I_{a}(x)+I_{m}(x)\right)} d x
$$

where $K$ stands for the spring composite stiffness, $E$ stands for the material elastic modulus, $\xi$ is the distortion correction coefficient, $I_{a}(x)$ is the sectional moment of inertia of the auxiliary spring, $I_{m}(x)$ is the sectional moment of inertia of the main spring, and $l_{m}$ stands for half-length of the main spring.

The integral equation is difficult to use in the direct calculation of the composite stiffness of the spring because of the variable cross sections of the main and auxiliary springs. Numerical integration is used to solve the problem, which cannot be performed without a computer. This study is conducted to determine whether a simple and accurate formula can be derived to calculate the composite stiffness of a parabolic leaf spring with variable stiffness. The theory of material mechanics is used to derive the equation for calculating the composite stiffness.

3.2. Derivation of Equation for Calculating the Deflection of a Mono-Leaf Spring. The deflection of the single parabolic leaf spring (mono-leaf spring) is the basis for calculating the deflection of a double-leaf spring. First, the mono-leaf spring equation is theoretically derived to obtain the equation for the deflection of any point along the leaf. The deformation of the leaf is considered when a force is applied at the end or middle part of the leaf. However, the leaf forced at the end is a special case compared with that when the leaf is forced at the 


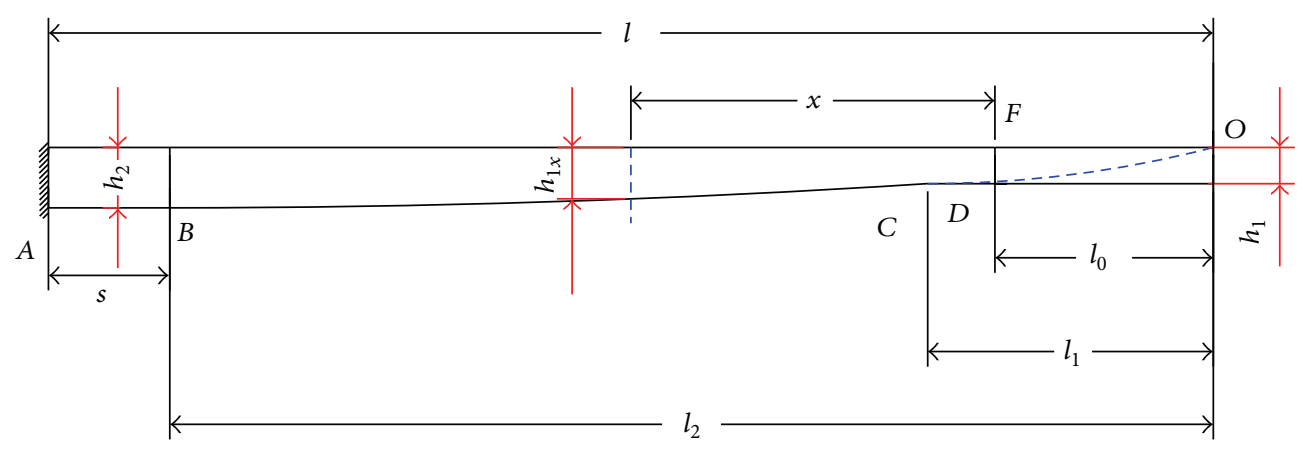

FIGURE 5: Mono-leaf spring (half-spring) with a force at its midsection.

midsection. First, the mono-leaf spring with an applied force at its midsection is considered.

3.2.1. Theoretical Derivation of Mono-Leaf Spring with an Applied Force at Its Midsection. Differential equations are established segmentally, and integration constants are obtained from boundary conditions on the basis of materialbend deformation theory. A force model of a mono-leaf spring (half-spring) with an applied force at its midsection is built and shown in Figure 5. Point $D$ is selected as the coordinate origin. The $x$-axis is located in the horizontal right, whereas the $y$-axis is vertically downward. $f(x)$ is the deflection of the point with a distance of $x$ from point $D . h_{1 x}$ is the thickness function of the parabolic part on the leaf, and $h_{1 x}=h_{2} \sqrt{\left(x+l_{0}\right) / l_{2}}$.

(1) Differential equations are built segmentally using material-bend deformation theory.

Step 1. For CD section with bending moment $M\left(x_{1}\right)=$ $F x_{1}\left(0 \leq x_{1} \leq l_{1}-l_{0}\right)$ and sectional moment of inertia $I_{1}$, the deflection curve differential equation is expressed as follows:

$$
E I_{1} f_{1}^{\prime \prime}\left(x_{1}\right)=F x_{1}
$$

The equation is integrated once, and the double integral of $x_{1}$ is as follows:

$$
\begin{aligned}
& E I_{1} f_{1}^{\prime}\left(x_{1}\right)=\frac{1}{2} F x_{1}^{2}+C_{1}, \\
& E I_{1} f_{1}\left(x_{1}\right)=\frac{1}{6} F x_{1}^{3}+C_{1} x_{1}+D_{1} .
\end{aligned}
$$

Step 2. For the BC section with bending moment $M\left(x_{2}\right)=$ $F x_{2}\left(l_{1}-l_{0} \leq x_{2} \leq l_{2}-l_{0}\right)$ and sectional moment of inertia $I_{2 x}(x)=b h_{1 x}^{3} / 12=\left(b h_{2}^{3} / 12\right)\left(\left(x_{2}+l_{0}\right) / l_{2}\right)^{3 / 2}=I_{2}\left(\left(x_{2}+\right.\right.$ $\left.\left.l_{0}\right) / l_{2}\right)^{3 / 2}$; then the deflection curve differential equation is expressed as follows:

$$
\begin{aligned}
E I_{2} f_{2}^{\prime \prime}\left(x_{2}\right) & =M\left(x_{2}\right) \\
& =l_{2}^{3 / 2} F\left[\left(x_{2}+l_{0}\right)^{-1 / 2}-l_{0}\left(x_{2}+l_{0}\right)^{-3 / 2}\right] .
\end{aligned}
$$

Integrating will result in the following equation:

$$
\begin{aligned}
E I_{2} f_{2}^{\prime}\left(x_{2}\right)= & 2 F l_{2}^{3 / 2}\left[\left(x_{2}+l_{0}\right)^{1 / 2}+l_{0}\left(x_{2}+l_{0}\right)^{-1 / 2}\right] \\
& +C_{2}, \\
E I_{2} f_{2}\left(x_{2}\right)= & 4 F l_{2}^{3 / 2}\left[\frac{1}{3}\left(x_{2}+l_{0}\right)^{3 / 2}+l_{0}\left(x_{2}+l_{0}\right)^{1 / 2}\right] \\
& +C_{2} x_{2}+D_{2} .
\end{aligned}
$$

Step 3. For the $A B$ section with bending moment of $M(x)=$ $F x_{3}\left(l_{2}-l_{0} \leq x_{3} \leq l-l_{0}\right)$ and sectional moment of inertia of $I_{2}$, the deflection curve differential equation is as follows:

$$
E I_{2} f_{3}^{\prime \prime}\left(x_{3}\right)=F x_{3} \quad\left(l_{2}-l_{0} \leq x_{3} \leq l-l_{0}\right) .
$$

Integrating will yield the following equations:

$$
\begin{aligned}
& E I_{2} f_{3}^{\prime}\left(x_{3}\right)=\frac{1}{2} F x_{3}^{2}+C_{3}, \\
& E I_{2} f_{3}\left(x_{3}\right)=\frac{1}{6} F x_{3}^{3}+C_{3} x_{3}+D_{3} .
\end{aligned}
$$

(2) Deflection and rotation angle are determined based on the boundary conditions and continuity of deflection to solve the constants in the differential equations.

Step 1. For the boundary condition in which the spring is fixed at point $C\left(x_{3}=l-l_{0}\right)$,

$$
f_{3}^{\prime}\left(l-l_{0}\right)=f_{3}\left(l-l_{0}\right)=0 .
$$

Constants $C_{3}$ and $D_{3}$ are calculated by (7)-(8) as follows:

$$
\begin{aligned}
& C_{3}=-\frac{F}{2}\left(l-l_{0}\right)^{2}, \\
& D_{3}=\frac{F}{3}\left(l-l_{0}\right)^{3} .
\end{aligned}
$$

Step 2. The continuity of deformation at point $B\left(x_{2}=x_{3}=\right.$ $l_{2}-l_{0}$ ) is considered:

$$
\begin{aligned}
& f_{2}^{\prime}\left(l_{2}-l_{0}\right)=f_{3}^{\prime}\left(l_{2}-l_{0}\right), \\
& f_{2}\left(l_{2}-l_{0}\right)=f_{3}\left(l_{2}-l_{0}\right) .
\end{aligned}
$$




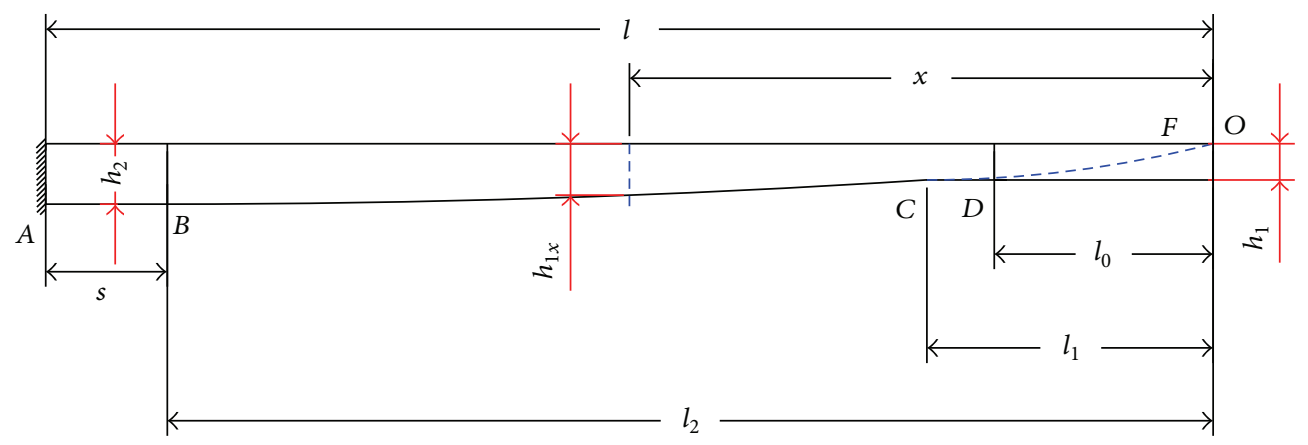

FIGURE 6: Mono-leaf spring (half-spring) with applied force at its end.

Constants $C_{2}$ and $D_{2}$ are calculated by (5), (7), and (10), as follows:

$$
\begin{aligned}
& C_{2}=F\left(-\frac{3}{2} l_{2}^{2}-3 l_{0} l_{2}+\frac{1}{2} l_{0}^{2}\right)+C_{3}, \\
& D_{2}=F\left(\frac{1}{3} l_{2}^{3}-3 l_{0} l_{2}^{2}-3 l_{0}^{2} l_{2}+\frac{1}{3} l_{0}^{3}\right)+D_{3} .
\end{aligned}
$$

Step 3. The continuity of deformation at point $B\left(x_{1}=x_{2}=\right.$ $\left.l_{1}-l_{0}\right)$ is considered. Thus,

$$
\begin{aligned}
& f_{1}^{\prime}\left(l_{1}-l_{0}\right)=f_{2}^{\prime}\left(l_{1}-l_{0}\right), \\
& f_{1}\left(l_{1}-l_{0}\right)=f_{2}\left(l_{1}-l_{0}\right) .
\end{aligned}
$$

Constants $C_{1}$ and $D_{1}$ are calculated by (3), (5), and (12), as follows:

$$
\begin{aligned}
C_{1} & =F\left[-\frac{3}{2} \alpha^{3}(1-\alpha) l_{2}^{2}-3 \alpha^{2}(\alpha-1) l_{0} l_{2}\right. \\
& \left.+\frac{1}{2}\left(\alpha^{3}-1\right) l_{0}^{2}\right]+C_{3} \alpha^{3}, \\
D_{1} & =F\left[\frac{1}{3} \alpha^{3}\left(1-\alpha^{3}\right) l_{2}^{3}-3 \alpha^{3}(1-\alpha) l_{0} l_{2}^{2}\right. \\
& \left.-3 \alpha^{2}(\alpha-1) l_{0}^{2} l_{2}+\frac{1}{3}\left(\alpha^{3}-1\right) l_{0}^{3}\right]+D_{3} \alpha^{3} .
\end{aligned}
$$

(3) The deflection curve function of the $C D$ section $(0 \leq$ $\left.x_{1} \leq l_{1}-l_{0}\right)$ is as follows:

$$
E I_{1} f\left(x_{1}\right)=\frac{1}{6} F x_{1}^{3}+C_{m 1} x_{1}+D_{m 1}
$$

where $C_{m 1}=C_{1} / F, D_{m 1}=D_{1} / F$.

The deflection and the rotation angle at point $D$ are obtained by setting $x_{1}$ to zero:

$$
\begin{aligned}
& f_{D 2}=f_{1}\left(x_{2}=0\right)=\frac{D_{m 1} F}{E I_{1}}, \\
& f_{D 2}^{\prime}=\frac{C_{m 1} F}{E I_{1}} .
\end{aligned}
$$

The deflection at point $O$ is as follows:

$$
f_{\mathrm{O} 2}=\frac{F\left(-C_{m 1} l_{0}+D_{m 1}\right)}{E I_{1}} .
$$

3.2.2. Theoretical Derivation of Mono-Leaf Spring with Applied Force at Its End. A force model of a mono-leaf spring (halfspring) with an applied force at its end is shown in Figure 6. This model is a special case of the model shown in Figure 5.

Thus, (14) can be used, and $l_{0}$ in (14) is set to zero to obtain the deflection curve function of CO section $\left(0 \leq x \leq l_{1}\right)$ as follows:

$$
\begin{aligned}
E I_{1} f(x)= & \frac{1}{6} F x^{3}+F\left[-\frac{l^{2}}{2} \alpha^{3}-\frac{3}{2}(1-\alpha) \alpha^{3} l_{2}^{2}\right] x \\
& +F\left[\frac{1}{3} l^{3} \alpha^{3}+\frac{1}{3} \alpha^{3}\left(1-\alpha^{3}\right) l_{2}^{3}\right],
\end{aligned}
$$

defining $D_{m 10}=\left(\alpha^{3} / 3\right)\left[l^{3}+\left(1-\alpha^{3}\right) l_{2}^{3}\right]$ and $C_{m 10}=\alpha^{3}\left[-l^{2} / 2-\right.$ $\left.(3 / 2)(1-\alpha) l_{2}^{2}\right]$.

Thus, the deflection at the end $(x=0)$ is as follows:

$$
f_{\mathrm{O} 1}=\frac{F D_{m 10}}{E I_{1}}
$$

The deflection at point $D\left(x=l_{0}\right)$ is expressed as follows:

$$
f_{D 1}=\frac{l_{0}^{3} / 6+D_{m 10}+C_{m 10} l_{0}}{E I_{1}} F .
$$

Thus, the deflection equations for a leaf with applied forces at the end and midsection are derived. These equations will be used for the calculation of the deflection of the doubleleaf spring.

3.3. Derivation of Equation for Composite Stiffness for DoubleLeaf Spring. A force model of a double-leaf spring (halfspring) with applied force at its end is shown in Figure 7. F and $F_{1}$ represent the forces applied at the end of the main and auxiliary springs, respectively. $F_{1}^{\prime}$ is the reaction force of $F_{1}$, and the deflection at point $O$ is a superposition of the deflection caused by $F_{1}^{\prime}$ and $F_{1}$. The force $F_{1}^{\prime}$ is initially determined using the boundary condition at point $D$. Thereafter, the deflection at point $O$ is calculated using the superposition principle of displacement. Finally, the composite stiffness of the double-leaf spring is obtained.

(1) Calculation of the Force at the Contact Point. The auxiliary spring has contact with the main spring only at its end when 


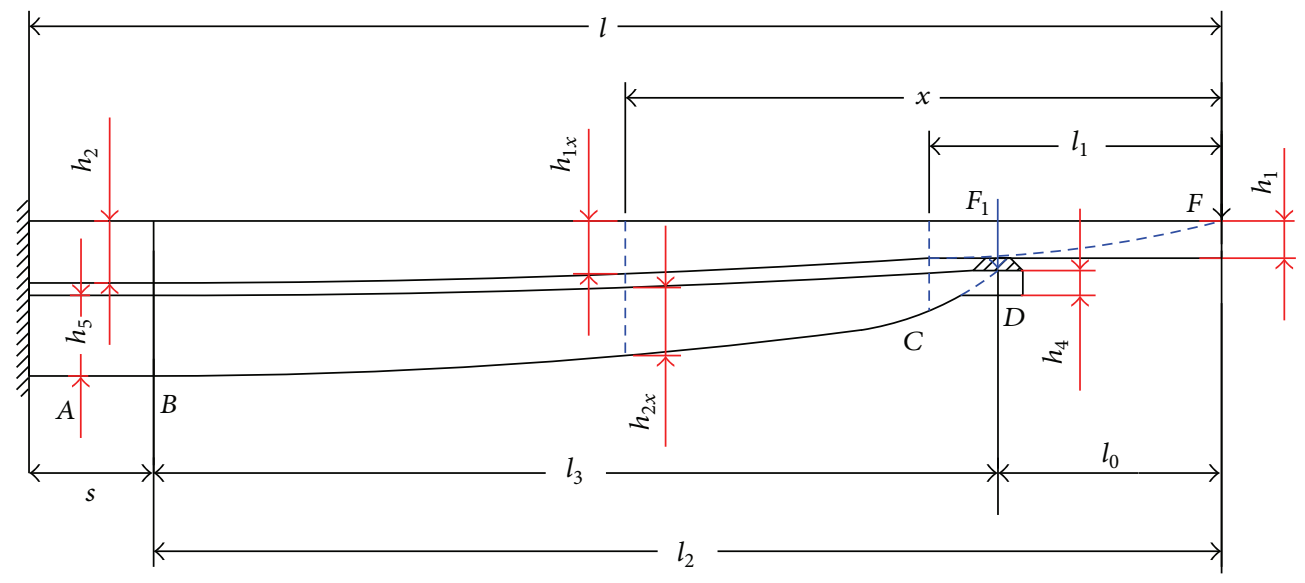

FigURE 7: Force model of a double-leaf spring (half-spring).

a force is applied at the end of a double-leaf spring. The force between the two leaves is transmitted through the contact point. The deflection and rotation angle between two leaves at the contact point do not remarkably vary. Thus, the contact point has two boundary conditions, namely, equal deflections and equal rotation angles. For each boundary condition, the force between the two leaves can be calculated.

Step 1 (the two leaves have the same deflections at the contact point). By using (18) and (19), the deflection at point $D$ of the main spring caused by force $F$ is as follows:

$$
f_{m D 1}=\frac{l_{0}^{3} / 6+D_{m 10}+C_{m 10} l_{0}}{E I_{1}} F .
$$

The deflection at point $D$ of the main spring caused by force $F_{1}^{\prime}$ is as follows:

$$
f_{m D 2}=\frac{F_{1} D_{m 1}}{E I_{1}} .
$$

The total deflection at point $D$ of the main spring is as follows: $f_{m D}=f_{m D 1}-f_{m D 2}$.

The deflection at point $D$ of the auxiliary spring caused by $F_{1}$ is as follows:

$$
f_{a D}=\frac{F_{1} D_{a 10}}{E I_{4}}
$$

where $D_{a 10}=\left(\beta^{3} F / 3\right)\left[\left(l-l_{0}\right)^{3}+\left(1-\beta^{3}\right) l_{3}^{3}\right]$.

For $f_{m D}=f_{a D}$, the force at the contact point because of the same deflection is as follows:

$$
F_{1 f}=\frac{\left(l_{0}^{3} / 6+D_{m 10}+C_{m 10} l_{0}\right)}{\gamma^{3} D_{a 10}+D_{m 1}} F .
$$

Step 2 (the two leaves have the same rotation angles at the contact point). The force between two leaves can be easily calculated when they have the same rotation angles at the contact point

$$
F_{1 \theta}=\frac{l_{0}^{2} / 2+C_{m 10}}{\gamma^{3} C_{a 10}+C_{m 10}} F,
$$

where $C_{a 10}=\beta^{3}\left[-\left(l-l_{0}\right)^{2} / 2-(3 / 2)(1-\beta) l_{3}^{2}\right]$.
The deflections or rotation angles of the two springs at their contact area are not exactly the same because of the rubber between the main and auxiliary springs at their contact area. The force at the contact point is neither $F_{1 f}$ nor $F_{1 \theta}$. The force is a combined effect of the two forces. Thus, force $F_{1}$ at the contact point is assumed to be as follows:

$$
F_{1}=\omega F_{1 f}+(1-\omega) F_{1 \theta}
$$

where $\omega$ is a weight coefficient, with a value that ranges from zero to one.

\section{(2) The Deflection at the End Point O Is Calculated}

Step 1. The deflection caused by force $F$ is expressed as follows:

$$
f_{\mathrm{O} 1}=\frac{F D_{m 10}}{E I_{1}} .
$$

Step 2. The deflection caused by force $F_{1}$ is expressed as follows:

$$
f_{\mathrm{O} 2}=\frac{F_{1}\left(D_{m 1}-C_{m 1} l_{0}\right)}{E I_{1}}
$$

Step 3. The total deflection at point $O$ is defined as follows:

$$
\begin{aligned}
f_{\mathrm{O}} & =f_{\mathrm{O} 1}-f_{\mathrm{O} 2}=\frac{F}{E I_{1}}\left\{D_{m 10}-\left(D_{m 1}-C_{m 1} l_{0}\right)\right. \\
& \cdot\left[\omega \frac{l_{0}^{3} / 6+D_{m 10}+C_{m 10} l_{0}}{\gamma^{3} D_{a 10}+D_{m 1}}\right. \\
& \left.\left.+(1-\omega) \frac{l_{0}^{2} / 2+C_{m 10}}{\gamma^{3} C_{a 10}+C_{m 10}}\right]\right\}=\frac{F \kappa_{\text {equ }} l^{3}}{E I_{1}},
\end{aligned}
$$




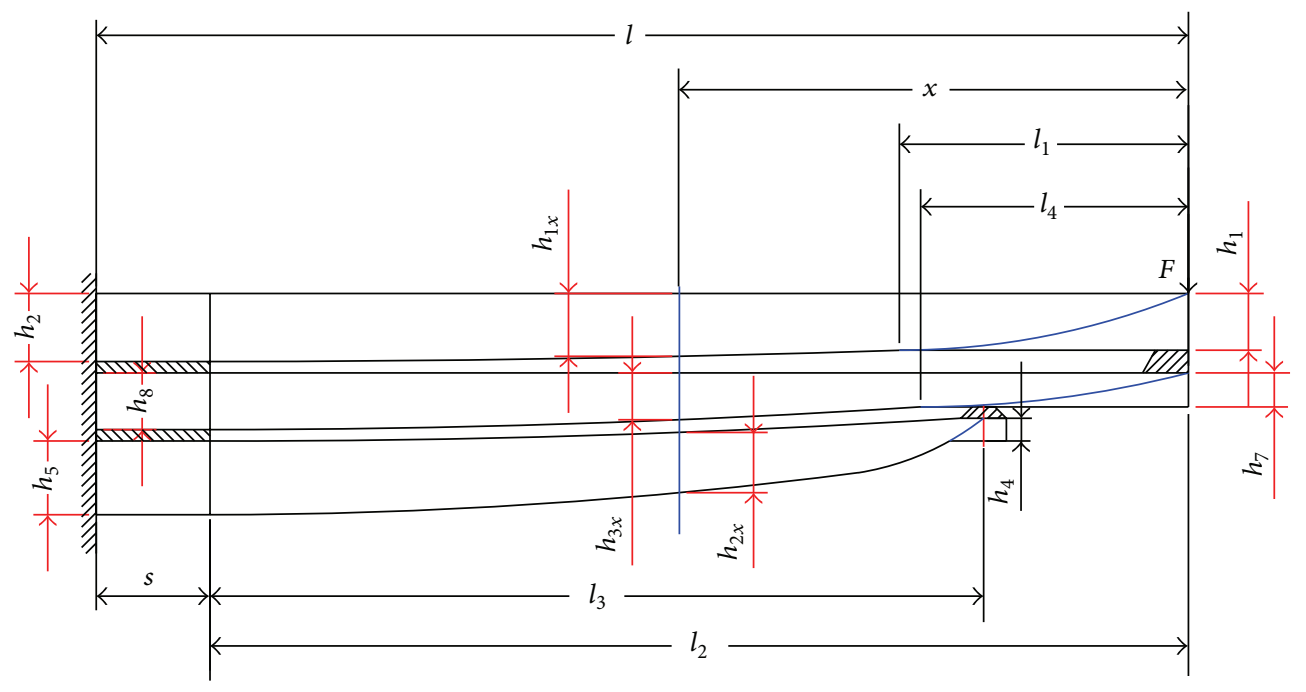

FIGURE 8: Force model of a triple-leaf spring (half-spring).

where

$$
\begin{aligned}
& \kappa_{\text {equ }}(\lambda, \mu, \alpha, \beta, \gamma)=d_{m 10}-\left(d_{m 1}-c_{m 1} \mu\right) \\
& \cdot\left[\omega \frac{(1 / 6) \mu^{3}+d_{m 10}+c_{m 10} \mu}{\gamma^{3} d_{a 10}+d_{m 1}}\right. \\
& \left.+(1-\omega) \frac{\mu^{2} / 2+c_{m 10}}{\gamma^{3} c_{a 10}+c_{m 1}}\right] \text {, } \\
& d_{m 10}=\alpha^{3} \frac{1+\left(1-\alpha^{3}\right) \lambda^{3}}{3} \text {, } \\
& c_{m 10}=-\alpha^{3} \frac{1+3(1-\alpha) \lambda^{2}}{2} \text {, } \\
& d_{m 1}=\frac{1}{3}(1-\mu)^{3} \alpha^{3}+\frac{1}{3} \alpha^{3}\left(1-\alpha^{3}\right) \lambda^{3}-3 \alpha^{3}(1-\alpha) \\
& \cdot \mu \lambda^{2}-3 \alpha^{2}(\alpha-1) \mu^{2} \lambda+\frac{1}{3}\left(\alpha^{3}-1\right) \mu^{3}=d_{m 10} \\
& -3 \alpha^{3}(1-\alpha) \mu \lambda^{2}-3 \alpha^{2}(\alpha-1) \mu^{2} \lambda-\frac{1}{3} \mu^{3}+\alpha^{3} \mu^{2} \\
& -\alpha^{3} \mu \\
& c_{m 1}=-\frac{(1-\mu)^{2}}{2} \alpha^{3}-\frac{3}{2}(1-\alpha) \alpha^{3} \lambda^{2}-3(\alpha-1) \alpha^{2} \mu \lambda \\
& +\frac{1}{2}\left(\alpha^{3}-1\right) \mu^{2}=c_{m 10}-3(\alpha-1) \alpha^{2} \mu \lambda-\frac{1}{2} \mu^{2} \\
& +\alpha^{3} \mu \text {. }
\end{aligned}
$$

Finally, an equation for calculating the composite stiffness of a double parabolic leaf spring is obtained, as follows:

$$
K_{\mathrm{two}}=\frac{F}{f_{\mathrm{O}}}=\frac{E I_{1}}{\kappa_{\mathrm{equ}} l^{3}} .
$$

Two methods are used to calculate the composite stiffness of a parabolic double-leaf spring to further refine the value range of $\omega$ in the equations. First, (30) is used to calculate directly, whereas the second approach is to use (1) and perform numerical integration to obtain the composite stiffness. The results from the two methods are compared when $\omega$ is set to different values. When $\omega$ is 0.5 to 0.7 , the result error between two methods is less than $5 \%$.

3.4. Derivation of an Equation for Calculating the Composite Stiffness of Triple-Leaf and Multileaf Springs. Compared with the double-leaf spring, triple- and multileaf springs have two or more main leaves with similar lengths. A simplified model is built as discussed in Section 2.2. Thus, the equation for calculating the composite stiffness of triple- and multileaf springs can be easily derived. A force model of a tripleleaf spring (half-spring) with an applied force at its end is built and shown in Figure $8 . h_{3 x}$ stands for the thickness of the auxiliary spring at the point with a distance of $x$ to the parabola vertex (end point of the main spring), $h_{3 x}=$ $h_{7} \sqrt{x / l_{2}} \cdot l_{4}$ stands for the length of the section with equal thickness at the end of the second main spring, $l_{4}=$ $l_{2}\left(h_{7} / h_{8}\right)^{2}$.

First, two main springs with different parameters are considered. The stiffness of the added main spring is expressed as follows:

$$
K_{s}=\frac{E I_{7}}{d_{2 m 10} l^{3}},
$$

where $d_{2 m 10}\left(\eta, \alpha_{2}\right)=\alpha_{2}^{3}\left(\left(1+\lambda^{3}\left(1-\alpha_{2}^{3}\right)\right) / 3\right), \alpha_{2}=h_{7} / h_{8}$, and $I_{7}=(1 / 12) b h_{7}^{3}$. follows:

The composite stiffness of a triple-leaf spring is defined as

$$
K_{\text {three }}=K_{\text {two }}+K_{s}=\frac{E I_{1}}{\kappa_{\text {equ }} l^{3}}+\frac{E I_{7}}{d_{2 m 10} l^{3}} .
$$


The equation for multileaf spring can be derived similarly. The parameters of the multi-main springs are generally the same. Thus, the equation for the composite stiffness of a triple-leaf spring can be simplified as follows:

$$
K_{\text {three }}=\frac{E I_{1}}{\kappa_{\text {equ }} l^{3}}+\frac{E I_{1}}{d_{m 10} l^{3}} .
$$

By contrast, the equation for the multileaf spring is expressed as follows:

$$
K_{h}=K_{\mathrm{two}}+(n-1) K_{t}=\xi\left[\frac{E I_{1}}{\kappa_{\mathrm{equ}} l^{3}}+\frac{(n-1) E I_{1}}{d_{m 10} l^{3}}\right],
$$

where $n$ stands for the number of the main springs and $\xi$ is a correction factor ranging from 0.92 to 0.99 .

\subsection{Calculation of Composite Stiffness of Taper-Leaf Spring} with Front and Rear Halves of Unequal Lengths. All previously derived equations for composite stiffness calculation were based on half-spring models. The composite stiffness of an entire leaf spring is calculated by determining the composite stiffness at the front and rear halves (usually they are not of equal lengths at the front and rear halves of the taper-leaf spring are are not equal just as the leaf-spring studied in this paper) which should be calculated first by using the equations shown above.

$K_{f}$ and $K_{r}$ represent the composite and the stiffness of the front and rear half springs of a taper-leaf spring. The composite stiffness of the entire spring can be calculated using (33):

$$
K=\left(K_{f}+K_{r}\right) \frac{\delta(1+\zeta)^{2}}{(1+\delta)\left(1+\delta \zeta^{2}\right)},
$$

where $\delta=K_{f} / K_{r}, \zeta=l_{f} / l_{r}$.

The composite stiffness of the front half spring is expressed as follows:

$$
K_{f}=\frac{E I_{1}}{\kappa_{\text {equ }} l_{f}^{3}}+\frac{E I_{1}}{d_{m 10} l_{f}^{3}} .
$$

The composite stiffness of the rear half spring is defined as follows:

$$
K_{r}=\frac{E I_{1}}{\kappa_{\text {equ }} l_{r}^{3}}+\frac{E I_{1}}{d_{m 10} l_{r}^{l}} .
$$

This calculation method is not limited to the triple-leaf spring studied in this paper. The method can also be used for multileaf springs.

3.6. Experimental and FEA Assessments. The correctness of the theoretical formula is verified by testing the mechanical properties of a fabricated triple-leaf spring (Figure 9). The two main leaves in the triple-leaf spring have similar geometric parameters, and the front and rear halves of each leaf have the same root thicknesses and end thicknesses. The geometric parameters of the triple-leaf spring are listed in Table 1.

\begin{tabular}{|c|c|c|c|c|}
\hline & Front & Rear & & \\
\hline \multirow[t]{3}{*}{$\begin{array}{l}\text { Length of } \\
\text { half-spring } \\
(\mathrm{mm})\end{array}$} & $l_{f}=692$ & $l_{r}=718$ & $\begin{array}{l}\text { Spring } \\
\text { width } b \\
(\mathrm{~mm})\end{array}$ & 60 \\
\hline & \multicolumn{2}{|c|}{ Main spring } & \multicolumn{2}{|c|}{ Auxiliary spring } \\
\hline & Front & Rear & Front & Rear \\
\hline $\begin{array}{l}\text { Length of } \\
\text { parabolic } \\
\text { portion }(\mathrm{mm})\end{array}$ & $l_{2 f}=633$ & $l_{2 r}=654$ & $l_{3 f}=492$ & $l_{3 r}=501$ \\
\hline $\begin{array}{l}\text { End thickness } \\
(\mathrm{mm})\end{array}$ & \multicolumn{2}{|c|}{$h_{1}=10.2$} & \multicolumn{2}{|c|}{$h_{4}=10.8$} \\
\hline $\begin{array}{l}\text { Root thickness } \\
(\mathrm{mm})\end{array}$ & \multicolumn{2}{|c|}{$h_{2}=14.2$} & \multicolumn{2}{|c|}{$h_{5}=23.4$} \\
\hline
\end{tabular}

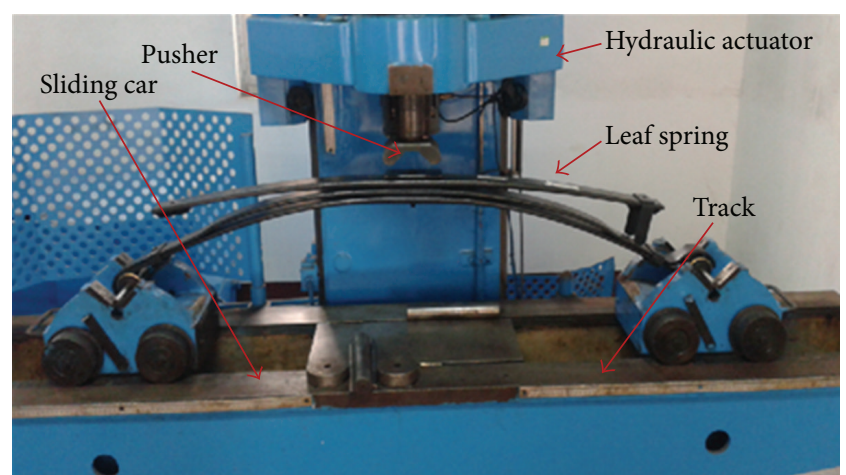

FIGURE 9: Experimental apparatus for measuring the stiffness of the triple-leaf spring.

TABLE 1: Geometric parameters of a triple-leaf spring.

The leaf spring is tested on a static stiffness test rig. The test rig consists of a hydraulic actuator, a pusher used to load the spring, rail base, tested leaf spring, sliding car, and so on. Two spring eyes are fixed to the sliding cars, and these eyes can only slide along the track when the spring is loaded. The leaf spring is loaded vertically by the actuator pusher. The load is gradually increased to $24.5 \mathrm{kN}$ from $0 \mathrm{~N}$ and then reloaded to $0 \mathrm{~N}$. The loading process, which is as long as the reloading process, is $120 \mathrm{~s}$ long. The load and displacement during testing are recorded, and their relationship is shown in Figure 10.

The experimental results show that hysteresis loss appears during the process of loading and reloading because of the friction between the main and auxiliary springs. Thus, a hysteresis loop is found in the displacement-force curve. The change trend of leaf spring stiffness with increasing load is similar to our predictions in the previous section. When the main and auxiliary springs are under full contact, the stiffness achieves a maximum and constant value.

The proposed formula is derived on the basis of the condition that the main and auxiliary springs are under full contact. Thus, the formula is only suitable for calculating the stiffness of the leaf spring when the main and auxiliary springs are under full contact. The simulation, experimental, and calculated equation (30) $(\omega=0.6)$ results are shown in Table 2. The error between the calculated and experimental 


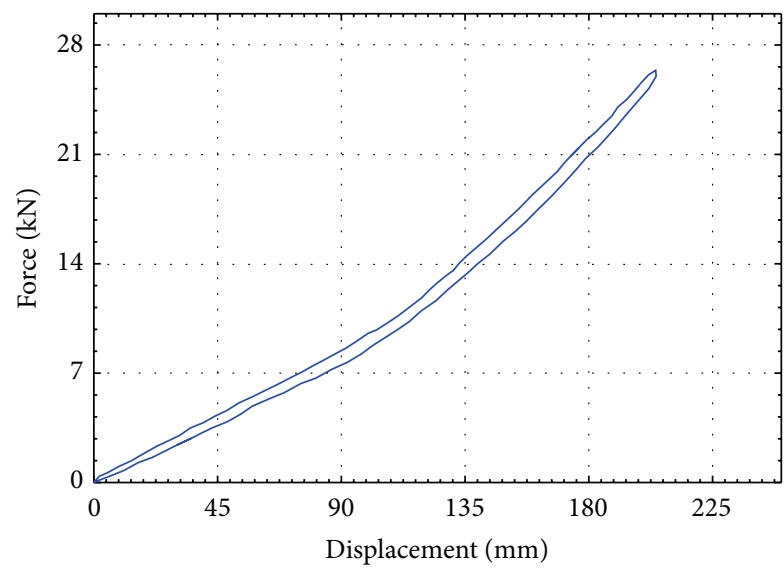

FIgURE 10: Stiffness of the triple-leaf spring.

TABLE 2: Comparative results.

\begin{tabular}{lcc}
\hline & Stiffness $(\mathrm{N} / \mathrm{mm})$ & $\begin{array}{c}\text { Relative error } \\
\text { with test result }\end{array}$ \\
\hline Test result & 183.8 & \\
Simulation result & 175.6 & $4.5 \%$ \\
Calculation result & 179.8 & $2.2 \%$ \\
\hline
\end{tabular}

results is small (within 5\%). The calculated value is smaller than the experimental value because the friction between the main and auxiliary springs is neglected during equation derivation process. Thus, the equation for composite stiffness calculation derived in this paper can fully meet the needs for engineering application. Moreover, the simulation result, which is close to the calculated result, verifies the correctness of the calculation equation.

\section{Attention of the Derived Equation in the Spring Design Process}

The correctness of the derived equation is confirmed by the results. Thus, the equation not only can be used to calculate the stiffness of existing leaf springs but also can be used in the design of a leaf spring. During the designing process, the geometric parameters of the spring leaves can be designed on the basis of the desired stiffness of the spring. However, in the actual machining process of the leaf spring, some actual dimensions of the spring leaf vary from the calculated dimensions when reliability, stress concentration, and other factors are considered. The effect of these differences on the composite stiffness value should be determined.

4.1. Root Thickness. The value of the root thickness of the leaf spring used in the formula is not equal to the one measured on a real leaf spring. This value is defined to be the vertical offset between the vertex of the parabolic leaf spring and the point in which the parabola reaches the U-bolt ( $h$ in Figure 11). However, in the production of a leaf spring, the root thickness of a leaf spring is designed to be equal to the vertical offset
TABLE 3: Geometric parameters of the mono-leaf spring model.

\begin{tabular}{lc}
\hline & Mono-leaf spring \\
\hline Spring width $b(\mathrm{~mm})$ & 80 \\
Length of half spring $l(\mathrm{~mm})$ & 800 \\
Length of parabolic portion $l_{2}(\mathrm{~mm})$ & 720 \\
End thickness $h_{1}(\mathrm{~mm})$ & 12 \\
Root thickness $h_{2}(\mathrm{~mm})$ & 25 \\
\hline
\end{tabular}

TABLE 4: Geometric parameters of mono-leaf spring model.

\begin{tabular}{lcc}
\hline $\begin{array}{l}\text { Length of half-spring } l(\mathrm{~mm}) \\
\text { Spring width } b(\mathrm{~mm})\end{array}$ & \multicolumn{2}{c}{700} \\
& Main spring & Auxiliary spring \\
\hline Length of parabolic portion $(\mathrm{mm})$ & $l_{2}=620$ & $l_{3}=500$ \\
End thickness $(\mathrm{mm})$ & $h_{1}=10$ & $h_{4}=20$ \\
Root thickness $(\mathrm{mm})$ & $h_{2}=8$ & $h_{5}=25$ \\
\hline
\end{tabular}

between the vertex of the spring leaf parabola and the point in which the parabola reaches the center bolt $(h+\Delta h$ in Figure 11) considering the reliability of the $\mathrm{U}$ bolt and reducing the stress of the spring at the U-bolt.

4.2. Transition Region between the Isopachous and Parabolic Portions of the Leaf Spring. During the actual processing of a leaf spring, the designer tends to increase at an arc transition region at the junction to reduce the stress concentration at the junction between isopachous and parabolic portions of the leaf spring (blue area in Figure 12). The finite element simulation analytical method is used because the shape of the transition region is difficult to use to describe the function.

The effect of this transition region on stiffness calculation is examined. A group of mono-leaf spring models and a group of double-leaf spring modes are used for FEA. Their geometric parameters are shown in Tables 3 and 4.

Both groups of models contain a model without a transition region and a model with arc transition region.

The transition region should not be too large or too small. It matches the size of the spring leaf (as shown in Table 5). Two groups of simulation results from ABAQUS are shown in Table 5. The existence of the transition region does not remarkably affect stiffness calculation, so the error of less than $2 \%$ can be neglected.

4.3. End Thickness of Auxiliary Leaf. The end thickness of an auxiliary leaf is the thickness of the uniform thickness at the end of the auxiliary leaf $\left(h_{4}\right.$ in Figure 1$)$. The auxiliary spring, not the main spring, generally bears only the vertical load. Thus, in the actual structure, its end thickness can be small enough to be close to zero. If a clip exists at the end of the auxiliary spring to transmit the lateral load or a rubber block to cushion stiffness mutation, the end thickness of the auxiliary spring is minimized as long as it satisfies certain needs of the lateral load and bearing reliability of the rubber block. This characteristic is also in line with the requirements of lightness of weight. 
TABLE 5: Simulation results of models with or without transition region.

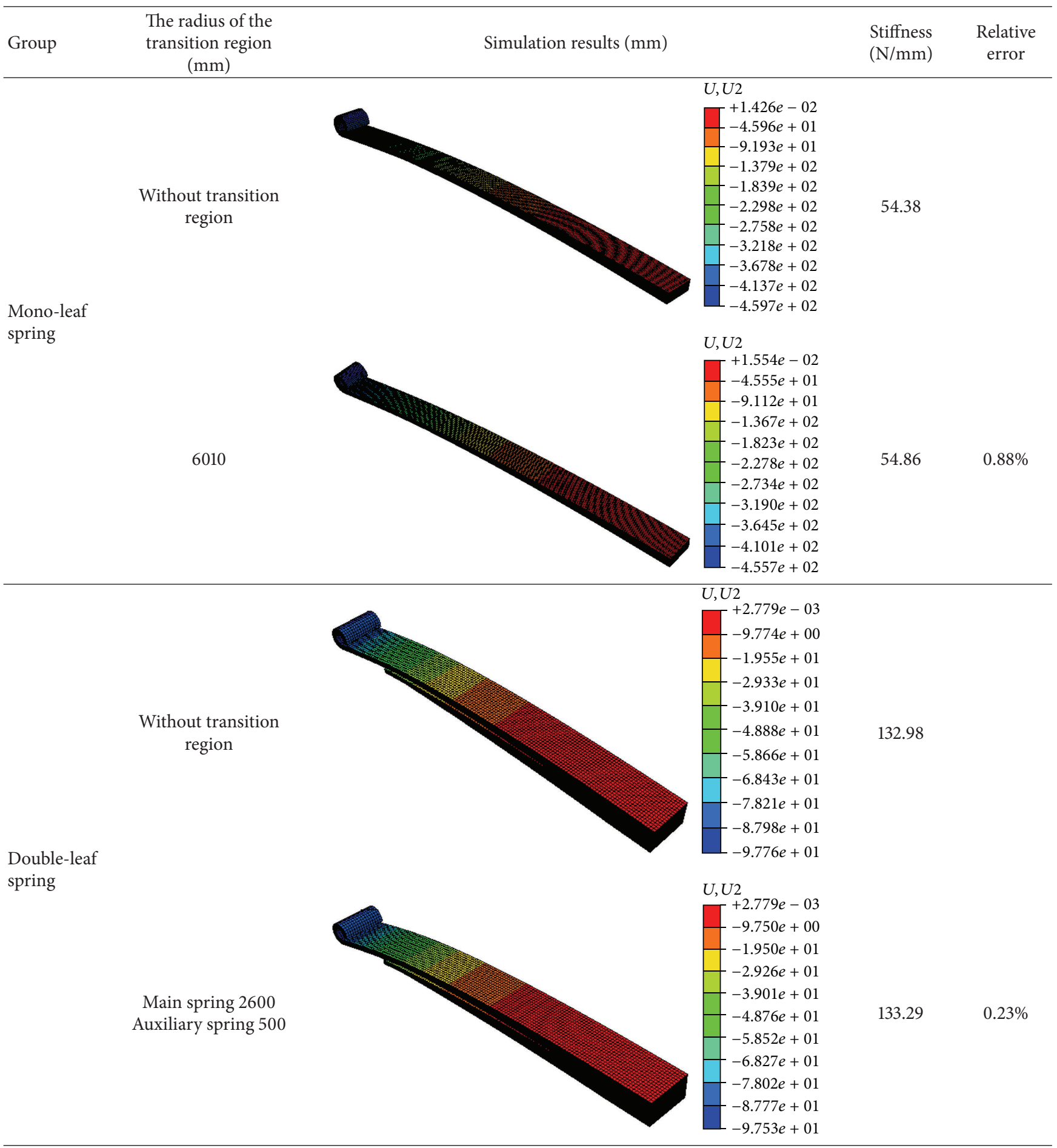

4.4. Other Factors. Other factors, such as U-bolt preload, leaf spring arc height, and surface treatment, also affect the stiffness value. The manner in which these factors affect the stiffness is similar to traditional leaf springs with uniform thickness.

\section{Conclusion}

The conclusion is as follows:

(1) An equation for calculating the composite stiffness for multileaf springs when the main and auxiliary spring 


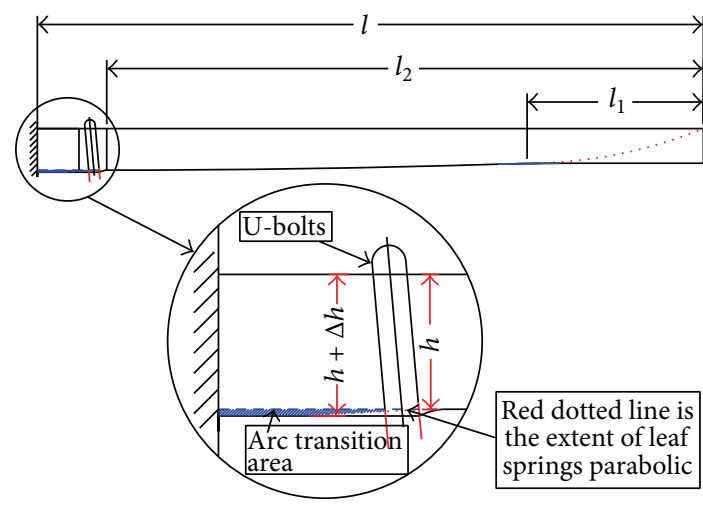

FIGURE 11: Root thickness of spring leaf.

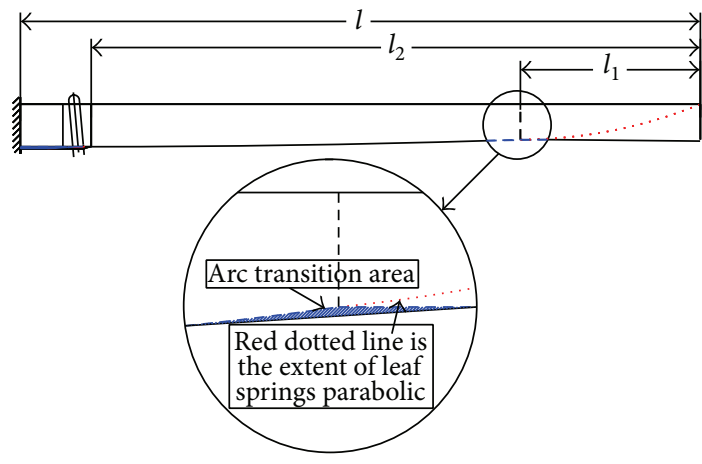

FIGURE 12: Transition region.

are under full contact is derived. The correctness of the calculation method is verified by the rig test and simulation.

(2) Parameters that should be considered for designing parabolic leaf springs are discussed to provide guidance for the design and manufacture of such leaf springs.

\section{Nomenclature}

$l$ : The length of the first main spring (subscripts $f$ and $r$ indicate the front half or rear half of the spring) (see $l_{f}$ and $l_{r}$ in Figure 1)

$l_{0}$ : The distance between the ends of the main and auxiliary springs (subscripts $f$ and $r$ indicate the front half or rear half of the spring) (see $l_{0 f}$ and $l_{0 r}$ in Figure 1)

$\mu$ : Ratio of $l_{0}$ to $l$

$l_{2}$ : The length of the parabolic portion of the first main spring (subscripts $f$ and $r$ indicate the front half or rear half of the spring) (see $l_{2 f}$ and $l_{2 r}$ in Figure 1)

$\lambda$ : Ratio of $l_{2}$ to $l$

$l_{3}$ : Length of the parabolic portion of the auxiliary spring (subscripts $f$ and $r$ indicate the front half or rear half of the spring) (see $l_{3 f}$ and $l_{3 r}$ in Figure 1) $l_{1}$ : $\quad$ Length of the isopachous portion of the first main spring (subscripts $f$ and $r$ indicate the front half or rear half of the spring) (see $l_{1 f}$ and $l_{1 r}$ in Figure 1)

$h_{1}, h_{2}, h_{3}$ : Front end thickness, root thickness, and rear end thickness of the first main spring

$\alpha: \quad$ Ratio of $h_{1}$ to $h_{2}$

$h_{4}, h_{5}, h_{6}: \quad$ Front end thickness, root thickness, and rear end thickness of the auxiliary spring

$\beta: \quad$ Ratio of $h_{4}$ to $h_{3}$

$\gamma: \quad \quad$ Ratio of $h_{1}$ to $h_{4}$

$h_{7}, h_{8}: \quad$ Root thickness and rear end thickness of the second main spring

$h_{1 x}, h_{2 x}, h_{3 x}$ : Thickness functions of the cross section in the parabolic portion of the first main spring, second main spring, and auxiliary spring

F: $\quad$ Force acting at the end of the leaf spring (subscripts $f$ and $r$ indicate the front half or rear half of the spring) (see $F_{f}$ and $F_{r}$ in Figure 1)

$s: \quad$ The half-length of the isopachous portion at the spring root

$b$ : $\quad$ Spring width

$\xi: \quad$ Distortion correction coefficient

$I_{1}$ : $\quad$ Sectional moment of inertia at the end of the first main spring; consider $I_{1}=$ $b h_{1}^{3} / 12$

$I_{2}$ : Sectional moment of inertia at the root of the first main spring; consider $I_{2}=$ $b h_{2}^{3} / 12$

$I_{3}: \quad$ Sectional moment of inertia at the end of the auxiliary spring; consider $I_{3}=$ $b h_{3}^{3} / 12$

$I_{4}$ : $\quad$ Sectional moment of inertia at the root of the auxiliary spring; consider $I_{4}=$ $b h_{4}^{3} / 12$.

\section{Conflict of Interests}

The authors declare no conflict of interests regarding the publication of this paper.

\section{Acknowledgments}

The authors would like to thank the School of Automotive Engineering, Changchun, Jilin, China, and the National Natural Science Foundation of China for supporting the project (Grant no. 51205158).

\section{References}

[1] S. Kim, W. Moon, and Y. Yoo, "An efficient method for calculating the nonlinear stiffness of progressive multi-leaf springs," 
International Journal of Vehicle Design, vol. 29, no. 4, pp. 403422, 2002.

[2] M. Bakir, M. Siktas, and S. Atamer, "Comprehensive durability assessment of leaf springs with CAE methods," SAE Technical Papers 2014-01-2297, 2014.

[3] R. Liu, R. Zheng, and B. Tang, "Theoretical calculations and experimental study of gradually variable rigidity leaf springs," Automobile Technology, vol. 11, pp. 12-15, 1993.

[4] G. Hu, P. Xia, and J. Yang, "Curvature-force hybrid method for calculating properties of leaf springs with variable stiffness," Journal of Nanjing University of Aeronautics \& Astronautics, vol. 40, no. 1, pp. 46-50, 2008.

[5] T. Horibe and N. Asano, "Large deflection analysis of beams on two-parameter elastic foundation using the boundary integral equation method," JSME International Journal Series A: Solid Mechanics and Material Engineering, vol. 44, no. 2, pp. 231-236, 2001.

[6] D. K. Roy and K. N. Saha, "Nonlinear analysis of leaf springs of functionally graded materials," Procedia Engineering, vol. 51, pp. 538-543, 2013.

[7] G. Savaidis, L. Riebeck, and K. Feitzelmayer, "Fatigue life improvement of parabolic leaf springs," Materials Testing, vol. 41, no. 6, pp. 234-240, 1999.

[8] M. M. Shokrieh and D. Rezaei, "Analysis and optimization of a composite leaf spring," Composite Structures, vol. 60, no. 3, pp. 317-325, 2003.

[9] Y. S. Kong, M. Z. Omar, L. B. Chua, and S. Abdullah, "Stress behavior of a novel parabolic spring for light duty vehicle," International Review of Mechanical Engineering, vol. 6, no. 3, pp. 617-620, 2012.

[10] M. Soner, N. Guven, A. Kanbolat, T. Erdogus, and M. K. Olguncelik, "Parabolic leaf spring design optimization considering FEA \& Rig test correlation,” SAE Technical Paper 2011-012167, 2011.

[11] Y. S. Kong, M. Z. Omar, L. B. Chua, and S. Abdullah, "Explicit nonlinear finite element geometric analysis of parabolic leaf springs under various loads," The Scientific World Journal, vol. 2013, Article ID 261926, 11 pages, 2013.

[12] W. Liu, Automotive Design, Tsinghua University Press, Beijing, China, 2001.

[13] Editorial Board, The Design Part of the Automobile Engineering Manual, People's Communications Press, Beijing, China, 2001.

[14] Y. Zhang, H. Liu, and D. Wang, Spring Manual, Machinery Industry Press, Beijing, China, 2008. 


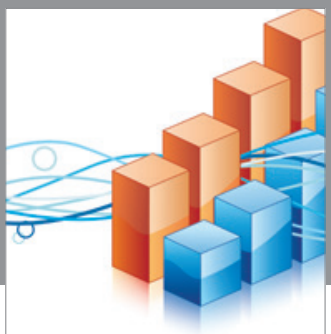

Advances in

Operations Research

vatem alat4

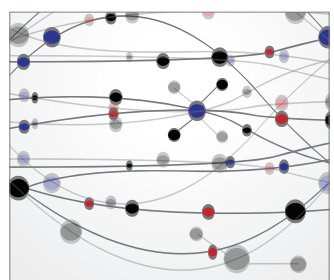

\section{The Scientific} World Journal
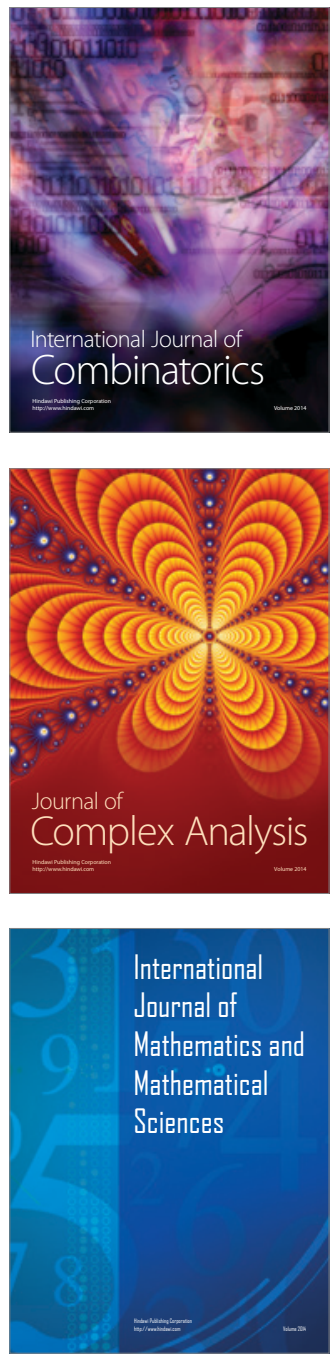
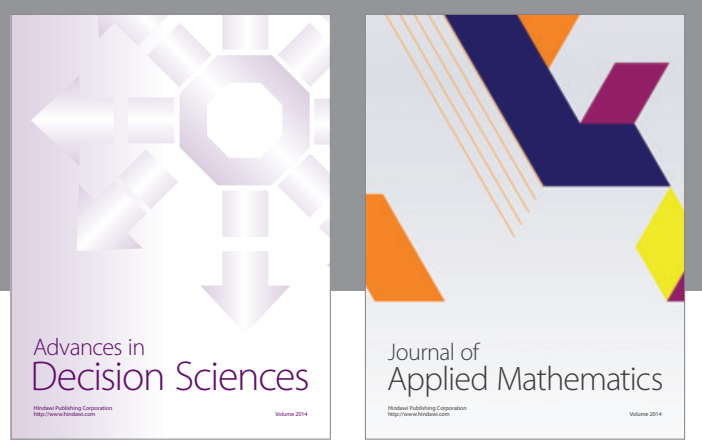

Algebra

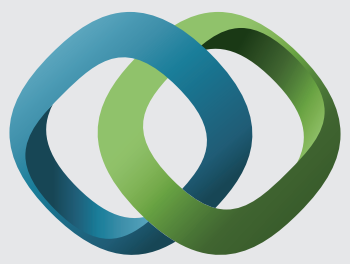

\section{Hindawi}

Submit your manuscripts at

http://www.hindawi.com
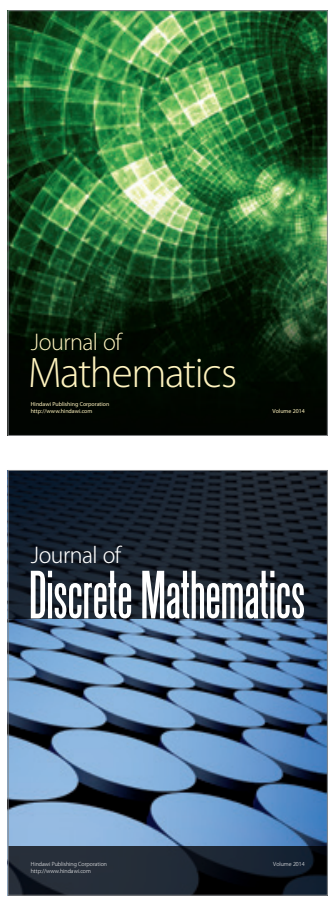

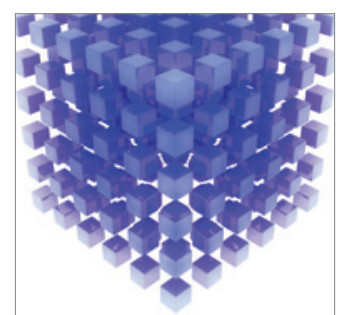

Mathematical Problems in Engineering
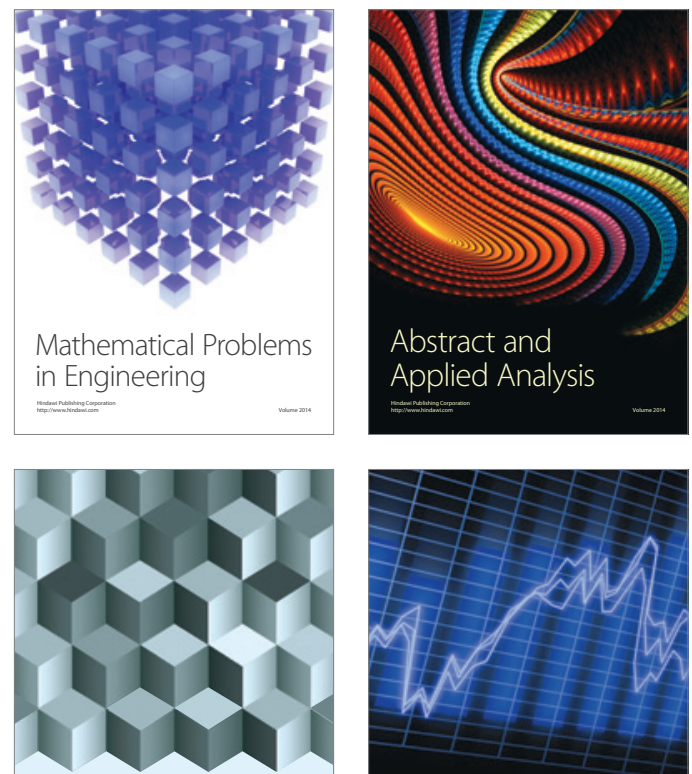

Journal of

Function Spaces

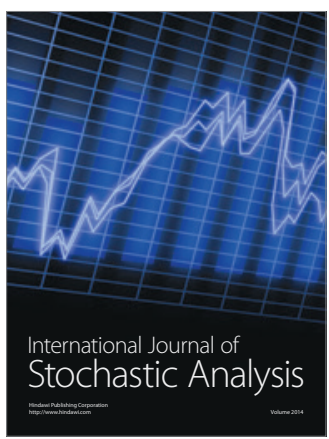

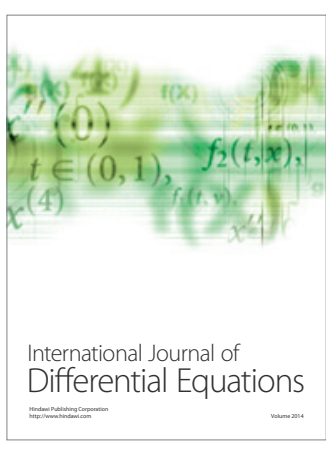
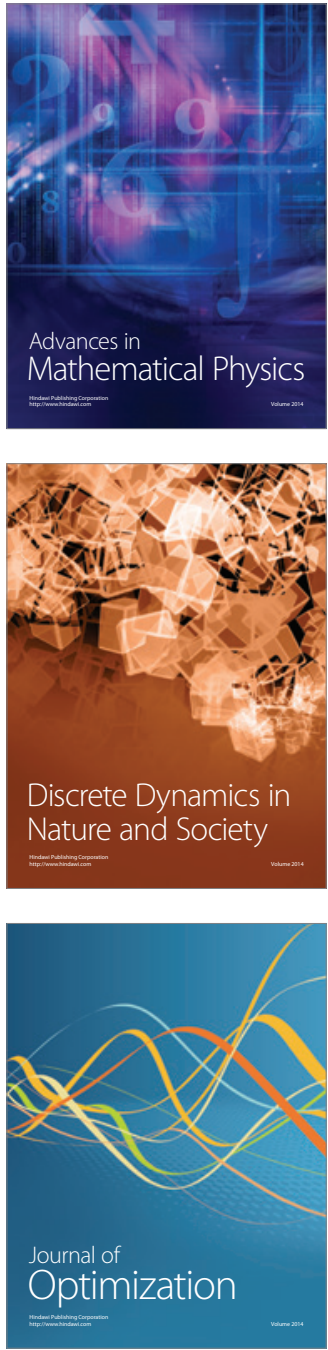